\title{
DISCONJUGATE LINEAR DIFFERENTIAL OPERATORS
}

\author{
BY \\ ZEEV NEHARI $\left({ }^{1}\right)$
}

In the present paper we study various properties of linear differential equations of the form

$$
L u \equiv p_{n}(x) L_{n} u+p_{n-1}(x) L_{n-1} u+\cdots+p_{0}(x) L_{0} u=0,
$$

where the operators $L_{k}$ are defined by

$$
L_{0} u=\rho_{1}(x) u, \quad L_{k} u=\rho_{k+1} \frac{d}{d x} L_{k-1} u, \quad k=1, \ldots, n .
$$

The functions $p_{0}(x), \ldots, p_{n}(x)$ and $\rho_{1}(x), \ldots, \rho_{n+1}(x)$ are assumed to be continuous in some open interval $(a, b)$, and the $\rho_{k}(x)$ as well as $p_{n}(x)$ are assumed to be positive. In order to ensure the solvability of the initial value problem at $x=a$, we also require all these functions to be bounded in a right neighborhood of $x=a$. The explicit form of $L_{k} u$ is

$$
L_{k} u=\rho_{k+1} \frac{d}{d x} \rho_{k} \frac{d}{d x} \cdots \frac{d}{d x} \rho_{1} u .
$$

The principal aim of this paper is the study of certain properties of equations of the special form

$$
L_{n} u=p(x) u, \quad p(x) \neq 0,
$$

where $p(x)$ is continuous in $(a, b)$, and properties of the general equation (1.1) will be derived only to the extent that they are necessary for this purpose.

A function $u(x)$ will be called a solution of (1) if $\rho_{1}(x) u(x)=y_{1}(x)$, and the functions $y_{1}(x), \ldots, y_{n}(x)$ are a set of continuous solutions of the first-order system of differential equations

$$
\begin{aligned}
& y_{k}^{\prime}=\frac{y_{k+1}}{\rho_{k+1}}, \quad k=1, \ldots, n-1, \\
& y_{n}^{\prime}=-\frac{1}{p_{n} \rho_{1} \rho_{n+1}}\left[p_{n-1} y_{n}+p_{n-2} y_{n-1}+\cdots+p_{0} y_{1}\right] .
\end{aligned}
$$

Under the assumptions made, (1.1) thus has precisely one solution $u(x)$ for which $L_{0} u, L_{1} u, \ldots, L_{n-1} u$ have given values at a point of $[a, b)$.

A differential equation $L u=0$ is said to be disconjugate in $(a, b)$-or, equivalently, the operator $L$ is said to be disconjugate in $(a, b)$-if no solution of $L u=0$ has more

Received by the editors January 18, 1966.

(1) This research was supported by the United States Air Force Office of Scientific Research under Grant AF-AFOSR 62-414. 
than $n-1$ zeros in $(a, b)$ (where $n$ is the order of $L$ ). In this definition, the zeros of a solution have to be counted with their multiplicities, and we say that $u(x)$ has a zero of order $m$ at a point $x=c$ if $L_{0} u=L_{1} u=\cdots=L_{m-1} u=0$ and $L_{m} u \neq 0$ for $x=c$. If $\rho_{k}(x) \equiv 1, k=1, \ldots, n+1$, i.e., if (1.1) reduces to the differential equation

$$
L u \equiv p_{n}(x) u^{(n)}+p_{n-1}(x) u^{(n-1)}+\cdots+p_{0}(x) u=0,
$$

then, according to a well-known result of Pólya [5], the equation is disconjugate in $(a, b)$ if, and only if, there exists a fundamental set of solutions $u_{1}, \ldots, u_{n}$ of (1.6) such that

$$
w_{k}>0, \quad k=1, \ldots, n-1, \quad x \in(a, b),
$$

where $w_{1}=u_{1}$ and the $w_{k}$ are the Wronskians

$$
w_{k}=\underset{v, \mu=1, \ldots, k}{\operatorname{det}}\left\{u_{v}^{(\mu-1)}\right\}, \quad k=2, \ldots, n-1 .
$$

An equivalent version of Pólya's result is that $L$ is disconjugate in $(a, b)$ if, and only if, $L u$ can be written in the form

$$
L u \equiv \rho_{n+1} \frac{d}{d x} \rho_{n} \frac{d}{d x} \cdots \frac{d}{d x} \rho_{1} u,
$$

where $\rho_{k}>0, k=1, \ldots, n+1$, and $\rho_{k}$ is of class $C_{n+1-k}$ in $(a, b)$. The equivalence of the two statements is a consequence of the identity

$$
L u \equiv \frac{w_{n}}{w_{n-1}} \frac{d}{d x} \frac{w_{n-1}^{2}}{w_{n-2} w_{n}} \frac{d}{d x} \cdots \frac{d}{d x} \frac{w_{2}^{2}}{w_{1} w_{3}} \frac{d}{d x} \frac{w_{1}^{2}}{w_{2}} \frac{d}{d x} \frac{u}{w_{1}}
$$

which goes back to Frobenius [1].

2. In what follows we shall require a generalization of Pólya's result which applies to equations of the type (1.1). As it stands, this result certainly is not valid, since the solutions of (1.1) need not be differentiable and the Wronskians (1.7) may not exist. We shall, however, show that the conclusion of Pólya's theorem remains true if suitable modifications are made. The main change necessary is the replacing of the determinants $(1.7)$ by the generalized Wronskians $W\left(u_{1}, \ldots, u_{k}\right)$ defined by

$$
W\left(u_{1}, \ldots, u_{k}\right)=\operatorname{det}_{v, \mu=1, \ldots, k}\left\{L_{v-1} u_{\mu}\right\}, \quad k=2, \ldots, n-1, \quad W\left(u_{1}\right)=L_{0} u_{1} .
$$

If the $u_{\mu}$ are solutions of (1.1), these Wronskians exist and are continuous.

In order to avoid excessive repetition we stipulate that, unless the contrary is stated explicitly, all statements made concerning functions of $x$ refer to the same interval $(a, b)$. For further reference, we now state the generalization of the two versions of Pólya's result in the form of two theorems.

THEOREM 2.1. Let $L$ denote the operator defined by (1.1) where the functions $\rho_{1}, \ldots, \rho_{n+1}, p_{0}, \ldots, p_{n}$ are continuous in $(a, b)$ and bounded near $x=a$, and let $\rho_{k}>0, k=1, \ldots, n+1$. In order that $L$ be disconjugate it is necessary and sufficient 
that there exist a fundamental set of solutions $u_{1}, \ldots, u_{n}$ of (1.1) for which the generalized Wronskians (2.1) are positive.

THEOREM 2.2. In order that the operator $L$ be disconjugate, it is necessary and sufficient that there exist positive and continuous functions $\sigma_{1}(x), \ldots, \sigma_{n+1}(x)$ such that the identity

$$
L_{n} \equiv \sigma_{n+1} \frac{d}{d x} \sigma_{n} \frac{d}{d x} \cdots \frac{d}{d x} \sigma_{1} u
$$

holds for any function $u(x)$ for which Lu exists and is continuous.

To prove these statements, we require the identity

$$
\frac{W_{k+1}^{2}}{\rho_{k+1} W_{k}} \frac{d}{d x}\left[\frac{W\left(u_{1}, u_{2}, \ldots, u_{k}, u\right)}{W_{k+1}}\right]=W\left(u_{1}, u_{2}, \ldots, u_{k+1}, u\right)
$$

where

$$
W_{r}=W\left(u_{1}, \ldots, u_{r}\right),
$$

and $u, u_{1}, \ldots, u_{k+1}$ are functions to which the operations $L_{0}, \ldots, L_{k+1}$ can be applied and lead to continuous functions. In order to avoid trivial cases, we also assume that the functions $u_{1}, \ldots, u_{k+1}$ are linearly independent. To establish (2.3), we note that, in view of the definition (2.1), the equation

$$
W\left(u_{1}, \ldots, u_{k+1}, u\right)=0
$$

may be regarded as a differential equation for $u$ with the fundamental set of solutions $u_{1}, \ldots, u_{k+1}$, and it is evident that (2.5) can be brought into the form

$$
W_{k+1} L_{k+1} u+\sum_{\nu=1}^{k} r_{v}(x) L_{v} u=0,
$$

where the coefficients $r_{v}(x)$ are continuous. On the other hand, the differential equation

$$
\frac{d}{d x}\left[\frac{W\left(u_{1}, \ldots, u_{k}, u\right)}{W_{k+1}}\right]=0
$$

also has the solutions $u_{1}, \ldots, u_{k+1}$, and (2.7) is easily seen to be of the form

$$
\frac{W_{k} \rho_{k+1}}{W_{k+1}} L_{k+1} u+\sum_{\nu=1}^{k} s_{\nu}(x) L_{\nu} u=0,
$$

where the $s_{v}(x)$ are again continuous. Hence, the differential equation

$$
\frac{W_{k+1}^{2}}{\rho_{k+1} W_{k}} \frac{d}{d x}\left[\frac{W\left(u_{1}, \ldots, u_{k}, u\right)}{W_{k+1}}\right]-W\left(u_{1}, \ldots, u_{k+1}, u\right)=0
$$

likewise has the linearly independent set of solutions $u_{1}, \ldots, u_{k+1}$. This, however, is absurd since, as shown by (2.6) and (2.8), (2.9) is an equation of order not exceeding $k$. It follows that the left-hand side of (2.9) must vanish identically, and this proves (2.3). 
With the abbreviations

$$
Y_{k}=\frac{W\left(u_{1}, \ldots, u_{k}, u\right)}{W_{k+1}}, \quad k=1, \ldots, n-1, \quad Y_{0}=\frac{u}{W_{1}}
$$

(2.3) may also be written

$$
\frac{W_{k+1}^{2}}{\rho_{k+1} W_{k} W_{k+2}} \frac{d Y_{k}}{d x}=Y_{k+1}
$$

and a repeated application of this identity makes it evident that

$$
W\left(u_{1}, \ldots, u_{n}, u\right) \equiv W_{n} \frac{d}{d x} \sigma_{n} \frac{d}{d x} \sigma_{n-1} \frac{d}{d x} \cdots \frac{d}{d x} \sigma_{1} u,
$$

where

$$
\sigma_{1}=\frac{1}{W_{1}}, \quad \sigma_{m}=\frac{W_{m-1}^{2}}{W_{m} W_{m-2} \rho_{m-1}}, \quad m=2, \ldots, n, \quad W_{0}=1
$$

The assumptions of the preceding analysis are satisfied if $u_{1}, \ldots, u_{n}$ are a fundamental set of solutions of (1.1) and if $u$ is any other solution of this equation. Since the equation $W\left(u_{1}, \ldots, u_{n}, u\right)=0$ has the same fundamental set, it follows from a comparison of the leading coefficients of these equations that

$$
L u \equiv \frac{\rho_{n+1}}{W_{n}} W\left(u_{1}, \ldots, u_{n}, u\right)
$$

(where, by classical results, $W_{n} \neq 0$ ). Hence, if we set

$$
\sigma_{n+1}=\frac{\rho_{n+1}}{W_{n}}
$$

the identity (2.2) is seen to be a consequence of (2.10) and (2.12). The functions $\sigma_{k}$ are defined by (2.11) and (2.13). The identity (2.2) represents the generalization of the Frobenius identity (1.8) to the case of operators of the form (1.1).

The equivalence of Theorems 2.1 and 2.2 is now an immediate consequence of (2.2). Indeed, the functions $W_{1}, \ldots, W_{n}$ are continuous (because of (1.5) and (1.3), they are even differentiable). If $W_{1}, \ldots, W_{n-1}$ are positive, and if we assume that $W_{n}>0$ (which can always be obtained by replacing $u_{n}$, if necessary, by $-u_{n}$ ), it thus follows from (2.11) and (2.13) that the functions $\sigma_{1}, \ldots, \sigma_{n+1}$ are positive and continuous. Conversely, if these functions have the latter properties, it is clear from (2.11) and (2.13) that the generalized Wronskians $W_{1}, \ldots, W_{n-1}$ are positive.

The fact that an operator with the decomposition (2.2) (with positive and continuous $\sigma_{k}$ ) is disconjugate, is elementary. Indeed, if $n$ is a nontrivial solution of $L u=0$, it follows from (2.2) that

$$
\frac{d}{d x} \sigma_{n-1} \frac{d}{d x} \cdots \frac{d}{d x} \sigma_{1} u=\frac{A}{\sigma_{n}}
$$

where $A$ is a constant. If $u$ has $n$ zeros, a repeated application of Rolle's theorem shows that the expression at the left-hand side vanishes at least once (we again 
remind the reader that all statements refer to the given interval $(a, b))$. This is impossible unless $A=0$. The equation thus reduces to

$$
\frac{d}{d x} \sigma_{n-2} \frac{d}{d x} \cdots \frac{d}{d x} \sigma_{1} u=\frac{B}{\sigma_{n-1}},
$$

where $B$ is a constant, and we again find that $B$ must vanish. Repeating this argument a sufficient number of times, we arrive at the absurd conclusion $\sigma_{1} u=$ const.

In order to complete the proof of Theorems 2.1 and 2.2, it remains to show that, in the case of a disconjugate equation (1.1), it is possible to choose a fundamental set of solutions $u_{1}, \ldots, u_{n}$ such that the generalized Wronskians $W_{1}, \ldots, W_{n-1}$ are all positive. To this end, we first remark that, under the assumptions imposed on the $\rho_{k}$ and $p_{k}$, the solutions of the system (1.5) have continuous extensions to the interval $[a, b)$, and that there exists a set $y_{1}, \ldots, y_{n}$ of such extensions in $[a, b)$ for which $y_{k}(a)=c_{k}, k=1, \ldots, n$, where the $c_{k}$ are arbitrary. Accordingly, we may define a fundamental set of solutions $u_{1}, \ldots, u_{n}$ of (1.1) by the initial conditions.

$$
\left.L_{n-\nu} u_{\mu}\right|_{x=a}=\delta_{v \mu}, \quad \nu, \mu=1, \ldots, n .
$$

Suppose now that one of the generalized Wronskians (2.1), say $W_{k}$, formed with these solutions vanishes for $x=c$, where $a<c<b$. This implies the existence of constants $A_{1}, \ldots, A_{k}$, not all zero, such that the solution $u=A_{1} u_{1}+\cdots+A_{k} u_{k}$ of (1.1) satisfies the conditions $L_{0} u=L_{1} u=\cdots=L_{k-1}=0$ at $x=c$. Hence, $u$ has a zero of order $k$ at $x=c$. Since, by (2.14), the solution $u_{\mu}$ has a zero of order $n-\mu$ at $x=a, u$ possesses a zero of order not less than $n-k$ at this point. The total number of zeros of $u$ in $[a, b)$ is thus found to be at least $n$. Because of the continuity of the solutions of the system (1.5) with respect to their initial values, this implies the existence of a solution of (1.1) with at least $n$ zeros in $(a, b)$. Since this contradicts our assumption that (1.1) is disconjugate, it follows that all the generalized Wronskians $W_{1}, \ldots, W_{n-1}$ must be different from zero. Replacing, if necessary, some of the functions $u_{k}$ by $-u_{k}$, we can make all these Wronskians positive. This completes the proof of Theorems 2.1 and 2.2.

In the case of an operator $L$ which is not disconjugate in $(a, b)$, special interest attaches to the conjugate point $x=\eta(a)$ of $x=a$, defined by $\eta(a)=\inf c$, where $a<c<b$ and $L$ is not disconjugate in $(a, c)$. As the preceding argument shows, the conjugate point has the following property (cf. [2], [3]):

THEOREM 2.3. If $x=\eta(a)$ is the conjugate point to $x=0$ with respect to the operator $L$, then there exists a solution $u$ of $L u=0$ which has a zero of order $m(1 \leqq m \leqq n-1)$ at $x=a$ and $a$ zero of order $n-m$ at $x=\eta(a)$.

We list here some other consequences of Theorem 2.2.

THEOREM 2.4. If $L$ and $R$ are disconjugate operators, the same is true of the operators $L R$ and $R L$. 
This follows from the fact that both $L$ and $R$ have representations of the form (2.2). Theorems 2.2 and 2.4 may also be summed up in the statement that an operator is disconjugate if and only if it can be decomposed into a product of disconjugate operators.

THEOREM 2.5. If an operator is disconjugate, so is its adjoint.

The adjoint $M$ of an operator $L$ is defined by the identity

$$
v L u-u M v=\frac{d}{d x} P(u, v)
$$

in $u$ and $v$, where $P(u, v)$ is a differential operation of order $n-1$ if $L$ is of order $n$. Since a disconjugate operator $L$ has the representation (2.2), it follows from (2.15) that

$$
M v \equiv(-1)^{n} \sigma_{1} \frac{d}{d x} \sigma_{2} \frac{d}{d x} \cdots \frac{d}{d x} \sigma_{n+1} v
$$

where $\sigma_{k}>0, k=1, \ldots, n+1$. Hence, $M$ is disconjugate. It may also be remarked that Theorem 2.5 is equivalent to the statement that the points conjugate to $x=a$ with respect to an operator and its adjoint coincide.

3. We now consider differential equations of the form (1.4). Since the behavior of the equation is very different according as the coefficient on the right-hand side of (1.4) is positive or negative, we shall write (1.4) in the form

$$
L_{n} u=\lambda p(x) u, \quad p(x)>0,
$$

where the positive function $p(x)$ is continuous and we have either $\lambda=1$ or $\lambda=-1$. We might of course also have absorbed the function $p(x)$ in the operator $L_{n}$ and replaced (3.1) by the equation

$$
L_{n} u=\lambda u .
$$

We also note that, because of Theorem $2.2, L_{n}$ may be taken to be an arbitrary disconjugate operator of the type (1.1).

Our basic result concerning equations of the form (3.1) is the following.

THEOREM 3.1. Let $u_{1}, \ldots, u_{n}$ be the fundamental set of solutions of (3.1) defined by the initial conditions

$$
\left.L_{v-1} u_{\mu}\right|_{x=a}=\delta_{v \mu}
$$

where the operators $L_{k}$ are given by (1.2), and denote by $W=W(x)$ the fundamental Wronskian matrix

$$
W=\left(L_{\nu-1} u_{\mu}\right), \quad \nu, \mu=1, \ldots, n .
$$

If $\lambda=-1$, all even-order minors of $W$ are positive in $(a, b)$; if $\lambda=1$, the same is true of all odd-order minors of $W$. 
The proof of this result will be based on the systematic utilization of a very elegant idea of Mikusiński [4], who applied it to the case of $2 \times 2$ Wronskians of solutions of the equation $u^{(n)}+p(x) u=0, p(x)>0$. For the minors of order $k$ we shall use the abbreviation

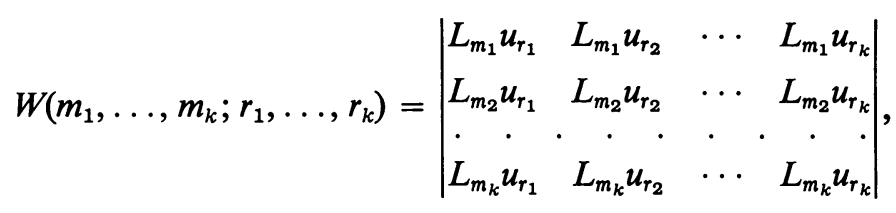

where

$$
0 \leqq m_{1}<m_{2}<\cdots<m_{k} \leqq n-1, \quad 1 \leqq r_{1}<r_{2}<\cdots<r_{k} \leqq n .
$$

Assuming first that $m_{k}<n-1$, we differentiate (3.5). Because of (1.2) and the rule for differentiating determinants, this yields

$$
\begin{aligned}
\frac{d}{d x} W & \left(m_{1}, \ldots, m_{k} ; r_{1}, \ldots, r_{k}\right) \\
& =\sum_{v=1}^{k} \frac{1}{\rho_{m_{v}+2}} W\left(m_{1}, \ldots, m_{v-1}, m_{v}+1, m_{v+1}, \ldots, m_{k} ; r_{1}, \ldots, r_{k}\right) .
\end{aligned}
$$

Whenever $k_{v+1}=k_{v}+1$, the corresponding determinants on the right-hand side of (3.7) will of course be zero. If $r_{k}=n-1$, then the last row of the last determinant in (3.7) will be composed of the terms $\rho_{n+1}^{-1} L_{n}\left(u_{r_{1}}\right), \ldots, \rho_{n+1}^{-1} L_{n}\left(u_{r_{k}}\right)$, or, because of (3.2), $\lambda \rho_{n+1}^{-1} u_{r_{1}}, \ldots, \lambda \rho_{n+1}^{-1} u_{r_{k}}$. Hence, a suitable permutation of the rows of this determinant shows that

$$
\begin{aligned}
\frac{d}{d x} W\left(m_{1}, \ldots, m_{k-1}, n-1 ; r_{1}, \ldots, r_{k}\right) & \\
= & \sum_{v=1}^{k-1} \frac{1}{\rho_{m_{v}+2}} W\left(m_{1}, \ldots, m_{v-1}, m_{v}+1, m_{v+1}, \ldots, m_{k} ; r_{1}, \ldots, r_{k}\right) \\
& +\frac{\lambda(-1)^{k-1}}{\rho_{n+1}} W\left(0, m_{1}, \ldots, m_{k}, r_{1}, \ldots, r_{k}\right)
\end{aligned}
$$

where the last determinant is again of the type (3.5) (and will be zero if $m_{1}=0$ ).

The relations (3.7) and (3.8) may be regarded as a system of first-order linear differential equations for the $W\left(m_{1}, \ldots, m_{k} ; r_{1}, \ldots, r_{k}\right)$, where $m_{1}, \ldots, m_{k}$ range over all the nonnegative integers satisfying the first condition (3.6). A glance at (3.7) and (3.8) shows that all the coefficients of this system are nonnegative when $\lambda=1$ and $k$ is odd, or when $\lambda=-1$ and $k$ is even. Hence, all solution components of the system will be nonnegative provided the initial values of all components at $x=a$ are nonnegative. Now the latter is indeed the case, since it is easily confirmed from (3.3) and (3.5) that the initial value of the expression (3.5) is 1 if $m_{v}=r_{v}-1$, $\nu=1, \ldots, k$, and is zero in all other cases. The nonnegativity of the coefficients of 
the system shows, moreover, that all solution components are nondecreasing, and it is not difficult to see that the identical vanishing of one solution component in an interval $(a, a+\varepsilon)$ would cause all the other components to vanish there. Since there exists one component which has the initial value 1 , this is impossible, and it follows that all components are positive for $x>a$. This completes the proof of Theorem 3.1.

We state here a few immediate consequences of Theorem 3.1.

THEOREM 3.2. If a nontrivial solution of (3.1) has zeros of order $k$ and $n-k$ at $x=a$ and $x=c$, respectively $(a<c<b)$, then $n-k$ is even or odd, according as $\lambda=1$ or $\lambda=-1$.

Indeed if a solution $u$ has a zero of order $k$ at $x=a$, we have

$$
u=A_{k+1} u_{k+1}+\cdots+A_{n} u_{n},
$$

where the $u_{v}$ are the solutions determined by (3.3) and the $A_{v}$ are constants. A zero of $u$ of order $n-k$ is thus equivalent to the vanishing of the minor

$$
W(0, \ldots, n-k-1 ; k+1, \ldots, n)
$$

of $W(x)$, and the result follows by an application of Theorem 3.1.

The preceding argument remains valid if the two multiplicities are assumed to be $\geqq k$ and $\geqq n-k$, respectively. This establishes the following assertion.

THEOREM 3.3. The sum of the multiplicities of two zeros of a solution of (3.1) cannot exceed $n$.

If they did, we could characterize these multiplicities as $\geqq k$ and $\geqq n-k$ or, alternatively, as $\geqq k-1$ and $\geqq n-k+1$. Clearly, one of these alternatives violates Theorem 3.2.

THEOREM 3.4. If $1 \leqq k \leqq n-2$, there exists-except for a multiplicative constantprecisely one nontrivial solution of (3.1) which has a zero of order $k$ at $x=a$ and $a$ zero of order $n-k-1$ at $x=c(a<c<b)$.

The existence of such a solution is elementary. To show the uniqueness, we suppose first that there are two such solutions, say $u$ and $v$, for which $k$ and $n-k-1$ are the precise orders of the zeros in question. We can then form a linear combination $A u+B v$ which has a zero of order $k+1$ at $x=a$ (and, of course, a zero of order $n-k+1$ at $x=c$ ). Similarly, there is a linear combination of $u$ and $v$ for which the orders of these zeros are $k$ and $n-k$, respectively. One of these linear combinations violates Theorem 3.2, unless it reduces to the trivial solution. Hence, $v=k u, k$ constant.

Next, suppose the precise order of the zero of $u$ at $x=c$ is $n-k$. By Theorem 3.3, the precise order of its zero at $x=a$ must be $k$. If these were also the orders of the zeros of $u$ at these points, we could form a solution $A u+B v$ which violates Theorem 3.3. Similarly, if the zeros of $v$ had the orders $k+1$ and $n-k-1$, respectively, 
either $u$ or $v$ would be in violation of Theorem 3.2. The precise orders of the zeros of $v$ must thus be $k$ and $n-k-1$. Hence, there exists a solution $A u+B v$ which has a zero of order $k+1$ at $x=a$ and a zero of order $n-k-1$ at $x=c$. However, since $u$ is a nontrivial solution with zeros of the orders $k$ and $n-k$ at $x=a$ and $x=c$, respectively, Theorem 3.2 shows that a contradiction can be avoided only if $A u+B v \equiv 0$.

4. In this section, we use Theorem 3.1 to prove the following result, which may be regarded as a generalization of the classical Sturm comparison theorem for second-order linear differential equations.

THEOREM 4.1. Let $L$ be an nth order differential operator of the form (1.1) and let $q_{1}(x)$ and $q_{2}(x)$ be continuous functions for which

$$
0 \leqq q_{1}(x) \leqq q_{2}(x) .
$$

Then, if both the equations $L u=0$ and $L u+q_{2} u=0$ are disconjugate, the equation $L u+q_{1} u=0$ is also disconjugate.

For the proof we require the following lemma.

LEMMA 4.1. Let $L_{k} u$ be the expressions defined by (1.2), and denote by $L_{k}^{*} u$ the corresponding expressions obtained if the functions $\rho_{1}, \ldots, \rho_{n+1}$ are replaced by functions $\sigma_{1}, \ldots, \sigma_{n+1}$. Let $W_{k}$ be the function (2.1) and $W_{k}^{*}$ the corresponding function obtained from the $L_{v}^{*}$. If $u$ is a function for which both $L_{k} u$ and $L_{k}^{*} u$ exist and are continuous, then

$$
\frac{W_{k}^{*}}{\prod_{v=1}^{k} \sigma_{v}^{k-v+1}}=\frac{W_{k}}{\prod_{v=1}^{k} \rho_{v}^{k-v+1}}
$$

The easiest way to verify this identity is by expressing the appearing derivatives as limits of suitable difference quotients, and then simplifying the obtained determinants before passing to the limit. We illustrate this process only for $k=2$; for higher $k$ it is entirely analogous. We have

$$
\begin{aligned}
& W_{2}=\lim _{x_{1} \rightarrow x}\left|\begin{array}{cc}
\rho_{1}\left(x_{1}\right) u_{1}\left(x_{1}\right) & \rho_{1}\left(x_{1}\right) u_{2}\left(x_{1}\right) \\
\rho_{2}(x)\left[\frac{\rho_{1}(x) u_{1}(x)-\rho_{1}\left(x_{1}\right) u_{1}\left(x_{1}\right)}{x-x_{1}}\right] & \rho_{2}(x)\left[\frac{\rho_{1}(x) u_{2}(x)-\rho_{1}\left(x_{1}\right) u_{2}\left(x_{1}\right)}{x-x_{1}}\right]
\end{array}\right| \\
& =\lim _{x_{1} \rightarrow x} \frac{\rho_{1}\left(x_{1}\right) \rho_{1}(x) \rho_{2}(x)}{x-x_{1}}\left|\begin{array}{ll}
u_{1}\left(x_{1}\right) & u_{2}\left(x_{1}\right) \\
u_{1}(x) & u_{2}(x)
\end{array}\right| .
\end{aligned}
$$

By comparing this with the corresponding expression for $W_{2}$ and letting $x_{1} \rightarrow x$, we find that

$$
\frac{W_{2}}{\rho_{1}^{2} \rho_{2}}=\frac{W_{2}^{*}}{\sigma_{1}^{2} \sigma_{2}}
$$


Turning now to the proof of Theorem 4.1, we note that the equation

$$
L u+q_{1} u=0 \quad\left(q_{1} \geqq 0\right)
$$

may also be written in the form

$$
M u-\left(q_{2}-q_{1}\right) u=0, \quad q_{2}-q_{1} \geqq 0,
$$

where $M$ is the differential operator $L+q_{2}$. By assumption, both $L$ and $M$ are disconjugate operators. Applying Theorem 3.1 (which clearly remains valid if the condition $p(x)>0$ in (3.1) is weakened to $p(x) \geqq 0, p(x) \not 0)$, we thus may conclude from (4.3) and (4.4) that both the even-order Wronskians $W_{k}$ (associated with $L$ ) and the odd-order Wronskians $W_{k}^{*}$ (associated with $M$ ) are positive. Since, by (4.2), $W_{k}$ and $W_{k}^{*}$ have the same sign, this shows that all the $W_{k}$ are positive. By Theorem 2.1, (4.3) is thus disconjugate. This proves Theorem 4.1. For selfadjoint fourth-order operators $L$, this result was obtained in [2]. For $n$th order operators $L$ of the special type (1.6), the theorem was stated (without proof) by A. Yu. Levin [3].

5. The term "disconjugate" was suggested by the fact that, in the case of a selfadjoint even-order equation, the possession of this property is equivalent to the absence in $(a, b)$ of a conjugate point to $x=a$ in the sense of Jacobi. It appears natural to introduce a companion concept which bears a similar relationship to the absence of a focal point in the classical case. We shall call equations (and operators) with this property "disfocal".

DEFINITION. The operator $L$ (defined in (1.1) and (1.2)) and the equation $L u=0$ are said to be disfocal in $(a, b)$ if, for any solution $u$ of $L u=0$, at least one of the functions $L_{0} u, \ldots, L_{n-1} u$ does not vanish in $(a, b)$.

It is clear that a disfocal operator is a fortiori disconjugate. Indeed, if a solution $u$ of $L u=0$ has $n$ zeros in $(a, b)$, a repeated application of Rolle's theorem shows that the functions $L_{1} u, \ldots, L_{n-1} u$ must all have zeros in $(a, b)$.

If the operator $L$ is not disfocal in $(a, b)$, there exists a "focal point" $x=\zeta(a)$ in $(a, b)$ characterized by $\zeta(a)=\inf c$ for all $c$ in $(a, b)$ such that $L$ is not disfocal in $(a, c)$. It is clear that $\zeta(a)>a$. If this were not the case, we could choose a sequence of solutions for which $c \rightarrow a$. Since this sequence can be normalized in such a way as to exclude the trivial limit $u \equiv 0$ (e.g., $A_{1}^{2}+\cdots+A_{n}^{2}=1$, where $u=A_{1} u_{1}+\cdots$ $+A_{n} u_{n}$ ), this leads to the absurdity of a nontrivial solution which satisfies the initial conditions $L_{0} u=L_{1} u=\cdots=L_{n-1} u=0$ at $x=a$.

In what follows we shall again confine ourselves to equations of the form

$$
L u \equiv L_{n} u-\lambda p(x) u=0, \quad p(x)>0, \quad \lambda= \pm 1 .
$$

For these equations, we establish the following analogues to Theorems 2.1 and 2.3.

THEOREM 5.1. Equation (5.1) is disfocal if and only if all principal minors of the fundamental Wronskian matrix are positive.

THEOREM 5.2. If $x=\zeta(a)$ is the focal point associated with $x=a$ with respect to equation (5.1), then there exist an integer $k(1 \leqq k \leqq n-1)$ and a solution $u(x)$ of 
(5.1) with the following properties: $k$ of the functions $L_{0} u, L_{1} u, \ldots, L_{n-1} u$ vanish at $x=a$, and the remaining $n-k$ at $x=\zeta(a)$. Except at the points indicated, the functions $L_{0} u, \ldots, L_{n-1} u$ are different from zero in $[a, \zeta(a)]$.

As a first step towards the proof of these theorems we establish two results, which may be regarded as analogues of Theorems 3.2 and 3.3.

THEOREM 5.3. Let $u$ be a solution of (5.1). If $k$ of the functions $L_{0} u, \ldots, L_{n-1} u$ vanish at $x=a$, and the remaining $n-k$ at $x=c(a<c<b, 1 \leqq k \leqq n-1)$, then $n-k$ is even or odd, according as $\lambda=1$ or $\lambda=-1$.

THEOREM 5.4. Let $u$ satisfy the hypotheses of Theorem 5.3. Then all the values of $L_{m} u(0 \leqq m \leqq n-1)$ at $x=a$ and $x=c$ which are not specifically assumed to be zero, are different from zero.

Theorem 5.3 is an immediate consequence of Theorem 3.1, since the existence of a solution with the indicated properties is equivalent to the vanishing at $x=c$ of a minor of order $n-k$ of the fundamental Wronskian matrix.

To establish Theorem 5.4, we first assume that $\lambda=1$ and note that, by Theorem $5.3, n-k$ must be even. If one of the $L_{m} u$ had an "extra" zero, say at $x=a$, then $u$ could be made to satisfy the hypotheses of Theorem 5.3 with $k+1$ of the functions $L_{0} u, \ldots, L_{n-1} u$ vanishing at $x=a$, and the remaining $n-k-1$ at $x=c$. Since $n-k+1$ is odd, this would violate the conclusion of Theorem 5.3. Similarly, an "extra" zero at $x=c$ would mean that $u$ satisfies the hypotheses of Theorem 5.3 with $k-1$ of the functions $L_{0} u, \ldots, L_{n-1} u$ vanishing at $x=a$, and the remaining $n-k+1$ at $x=c$. Again, $n-k+1$ is odd, contrary to Theorem 5.3. If $\lambda=-1$, the required modifications of the argument are obvious.

In the proof of Theorem 5.2 we shall employ a continuity argument which, for the sake of convenience, we state here as a lemma.

LEMMA 5.1. If the solution $u$ of (1.1) for which $n-1$ of the functions $L_{0} u, \ldots, L_{n-1} u$ vanish, respectively, at the points $x=x_{1}, x=x_{2}, \ldots, x=x_{n-1}$ is essentially unique (i.e., except for a constant factor), then $u=u\left(x ; x_{1}, \ldots, x_{n-1}\right)$ is a continuous function of $x_{1}, \ldots, x_{n-1}$ if properly normalized.

To make things definite, we suppose that $L_{k} u\left(x_{k}\right)=0, k=1, \ldots, n-1$. It is evident that a suitable definition of $u\left(x ; x_{1}, \ldots, x_{n-1}\right)$ is

$$
u\left(x ; x_{1}, \ldots, x_{n-1}\right)=\left|\begin{array}{cccc}
L_{1} u_{1}\left(x_{1}\right) & L_{1} u_{2}\left(x_{1}\right) & \ldots & L_{1} u_{n}\left(x_{1}\right) \\
L_{2} u_{1}\left(x_{2}\right) & L_{2} u_{2}\left(x_{2}\right) & \ldots & L_{2} u_{n}\left(x_{2}\right) \\
& & \vdots & \\
L_{n-1} u_{1}\left(x_{n-1}\right) & L_{n-1} u_{2}\left(x_{n-1}\right) & \cdots & L_{n-1} u_{n}\left(x_{n-1}\right) \\
u_{1}(x) & u_{2}(x) & \ldots & u_{n}(x)
\end{array}\right|,
$$

provided this expression does not vanish identically in $x$. The lemma will thus be proved if we can show that $u\left(x ; x_{1}, \ldots, x_{n-1}\right) \not \equiv 0$ if the solution $u$ is essentially unique. 
If $u\left(x ; x_{1}, \ldots, x_{n-1}\right) \equiv 0$, the minors of $u_{1}(x), \ldots, u_{n}(x)$ in the determinant (5.1) must all be zero. From the vanishing of the minor of $u_{1}(x)$ it follows that there exist constants $A_{2}, \ldots, A_{n}\left(A_{2}^{2}+\cdots+A_{n}^{2}>0\right)$ such that

$$
A_{2} L_{k} u_{2}\left(x_{k}\right)+A_{3} L_{k} u_{3}\left(x_{k}\right)+\cdots+A_{n} L_{k} u_{n}\left(x_{k}\right)=0
$$

for $k=1, \ldots, n-1$, i.e., (1.1) has a nontrivial solution $v_{1}=A_{2} u_{2}+\cdots+A_{n} u_{n}$ for which $L_{k} v_{1}\left(x_{k}\right)=0$ for $k=1, \ldots, n-1$. Similarly, the vanishing of the minor of $u_{r}(x)$ leads to the existence of a nontrivial solution $v_{r}$ which is a linear combination of $u_{1}, \ldots, u_{r-1}, u_{r+1}, \ldots, u_{n}$ and which likewise satisfies the homogeneous conditions imposed on $u$. If two of these solutions are essentially the same, i.e., $u_{r}=C u_{s}(r \neq s, C$ constant $)$, then the linear expression for $v_{r}$ in terms of the $u_{1}, \ldots, u_{n}$ cannot contain either $u_{r}$ or $u_{s}$. Applying the same argument to three or more solutions $v$, we find that not all the solutions $v_{1}, \ldots, v_{n}$ can be essentially the same, since this would lead to the absurd consequence that in the representation $B_{1} u_{1}+\cdots+B_{n} u_{n}$ of the nontrivial solution $v_{1}$ all the constants $B_{1}, \ldots, B_{n}$ must be zero. Hence there must exist two essentially different solutions $v$ which satisfy the homogeneous conditions imposed on $u$. Since this is excluded by our assumptions, we cannot have $u\left(x ; x_{1}, \ldots, x_{n-1}\right) \equiv 0$. This completes the proof of Lemma 5.1.

We now turn to the proof of Theorem 5.2. It follows from the definition of $\zeta(a)$ that there exists a solution $u$ of (1.1) for which each of the functions $L_{0} u, \ldots, L_{n-1} u$ vanishes somewhere in $[a, \zeta(a)]$. In case there exists more than one solution, we choose one-to be referred to as the "maximal" solution-for which the number $N$ of the functions $L_{0} u, \ldots, L_{n-1} u$ which vanish at either $x=a$ or $x=\zeta(a)$ is as large as possible. We wish to show that the assumption $N<n$ leads to a contradiction. With this aim in view, we first observe that a maximal solution with this property is uniquely determined (except for a constant factor) by the zeros (one each) of $n-1$ of the functions $L_{0} u, \ldots, L_{n-1} u$, provided the latter include the zeros at $x=a$ and $x=\zeta(a)$. Indeed, if there were two such solutions, we could form a linear combination for which an additional $L_{k} u$ vanishes at $x=a$ or $x=\zeta(a)$, and this would contradict the maximal character of $u$.

This uniqueness property of the maximal solution $u$ makes it possible to apply Lemma 5.1. We wish to show that a function $L_{m} u(0 \leqq m \leqq n-1)$ which vanishes neither at $x=a$ nor at $x=\zeta(a)$ cannot change its sign in $(a, \zeta(a))$. To do so, we assume that there is such a sign change at $x=c(a<c<\zeta(a))$, and we denote by $\alpha_{k}(k \neq m)$ one of the zeros of $L_{k} u(0 \leqq k \leqq n-1)$ in $[a, \zeta(a)]$ (where all the zeros of $L_{k} u$ at either $x=a$ or $x=\zeta(a)$ are included). Next, we construct a solution $v$ which satisfies the following conditions: $L_{k} v=0$ for $x=\beta_{k}, 0 \leqq k \leqq n-1, k \neq m$, where $\beta_{k}=\alpha_{k}$ for those $\alpha_{k}$ which do not coincide with $\zeta(a)$ and $\beta_{k}=\zeta(a)-\varepsilon(\varepsilon>0$, $a<\zeta(a)-\varepsilon)$ in the other cases. Except for a constant factor, these conditions determine $v$ uniquely; if there were two such solutions, we could evidently form a linear combination which fails to be disfocal in the interval $[a, \zeta(a)-\varepsilon]$, contrary to the definition of $\zeta(a)$. By Lemma 5.1, $v$ is thus a continuous function of $\varepsilon$ (if 
properly normalized). If $\varepsilon \rightarrow 0$, it follows from the uniqueness property of $u$ mentioned above that $v \rightarrow u$. Since $L_{m} u$ changes its sign at $x=c(a<c<\zeta(a))$, this shows that $L_{m} v$ must likewise change its sign at an interior point, provided $\varepsilon$ is taken small enough. For such values of $\varepsilon$, each of the functions $L_{0} v, \ldots, L_{n-1} v$ thus vanishes at some point of the interval $[a, \zeta(a)-\varepsilon]$. But this conflicts with the definition of the focal point, and we have thus proved that $L_{m} u$ cannot change its sign in $(a, \zeta(a))$ if $u$ is a maximal solution for which $L_{m} u \neq 0$ at $x=a, \zeta(a)$.

Since $L_{m} u$ must vanish somewhere in $[a, \zeta(a)]$, the assertion $N=n$ will be established if we can show the assumption that $L_{m} u \neq 0$ at $x=a, \zeta(a)$ is also not compatible with an even-order zero of $L_{m} u$ at $x=c(a<c<\zeta(a))$. The argument which we use for this purpose will also show, at the same time, that no $L_{k} u$ can vanish in $(a, \zeta(a))$. Suppose, then, that $L_{m} u$ has a zero in $(a, \zeta(a))$ at which it does not change its sign. At this point, $L_{m+1} u$ necessarily changes its sign. If $m=n-1,(5.1)$ shows that $L_{0} u$ has a change of sign at this point; to simplify the writing we therefore, in this context, replace $L_{r} u$ by $L_{r-n+1} u$ whenever $r>n-1$. Since $L_{m+1} u$ changes its sign in $(a, \zeta(a))$, it follows from what we have just proved that $L_{m+1} u$ must vanish at either $x=a$ or $x=\zeta(a) . L_{m+1} n$ thus has two distinct zeros in [a, $\left.\zeta(a)\right]$. By Rolle's theorem, $L_{m+2} u$ must therefore have a change of sign in $(a, \zeta(a))$ and thus, by our preceding result, $L_{m+2} u$ must vanish for either $x=a$ or $x=\zeta(a)$. By repeating this argument a sufficient number of times and observing our convention regarding the subscript of $L_{r} u$, we finally arrive at the conclusion that $L_{m} u$ changes its sign in $(a, \zeta(a))$, contrary to our assumption.

As pointed out above, this argument also produces a contradiction if we assume that, for some $m$ such that $0 \leqq m \leqq n-1, L_{m} u$ vanishes both in the interior and at a boundary point of the interval $[a, \zeta(a)]$. Hence, each $L_{m} u(m=0, \ldots, n-1)$ vanishes at one of the endpoints of the interval $[a, \zeta(a)]$ and is different from zero in the interior. Since, by Theorem 5.4, $L_{m} u$ cannot vanish at the other end of this interval, this completes the proof of Theorem 5.2.

Theorem 5.1 is an immediate consequence of these properties of the solution $u$. Since $L_{p_{\nu}} u=0$ for $x=a$ and $L_{r_{\mu}} u=0$ for $x=\zeta(a)$, where $\nu=1, \ldots, k, \mu=1, \ldots, n-k$, $p_{\nu} \neq r_{\mu}, 0 \leqq p_{v}, r_{\mu} \leqq n-1, u$ can be written in the form $u=A_{r_{1}} u_{r_{1}}+\cdots+A_{r_{n-k}} u_{r_{n-k}}$, where the $u_{m}$ are the solutions defined by (3.3) and not all the $A_{r}$ are zero. Substituting this expression in the conditions $L_{r_{\mu}} u=0, x=\zeta(a), \mu=1, \ldots, n-k$, we find that the principal minor $D\left(r_{1}, \ldots, r_{n-k} ; r_{1}, \ldots, r_{n-k}\right)$ of the fundamental Wronskian matrix must vanish at $x=\zeta(a)$. Conversely, if one of the principal minors vanishes at a point $x=c(a<c<b)$, this implies the existence of a solution $u$ for which all the functions $L_{0} u, \ldots, L_{n-1} u$ vanish at either $x=a$ or $x=c$. The equation thus fails to be disfocal in any interval $\left(a, c^{\prime}\right)$ for which $c^{\prime}>c$. Accordingly, the focal point coincides with the smallest zero of one of the principal minors in $(a, b)$.

6. According to Theorem 2.5, the disconjugacy of an operator implies that of the adjoint operator. The question whether an analogous result holds in general for 
disfocal operators seems to be more difficult to decide. As the following result shows, the answer is in the affirmative in the case of operators of the special form (5.1).

THEOREM 6.1. If an operator of the type (5.1) is disfocal, then the same is true of its adjoint operator.

The operator $M$ adjoint to $L$ is defined by

$$
M v \equiv(-)^{n} M_{n} v-\lambda p(x) v,
$$

where

$$
M_{0} v=\rho_{n+1} v, \quad M_{k} r=\rho_{n+1-k} \frac{d}{d x} M_{k-1} v, \quad k=1, \ldots, n .
$$

Integrating by parts and taking into account the relations (1.2) and (6.2), we obtain the Green's identity

$$
\begin{aligned}
\int_{a}^{c}(v L u-u M v) d x & =\int_{a}^{c}\left(v L_{n} u-(-1)^{n} u M_{n} v\right) d x \\
& =\left[M_{0} v L_{n-1} u-M_{1} v L_{n-2} u+-\cdots-(-1)^{n} M_{n-1} v L_{0} u\right]_{a}^{c}
\end{aligned}
$$

Suppose now that $L$ is disfocal in $(a, b)$, while $M$ is not. The operator $M$ will then possess a focal point with respect to $x=a$ at, say, $x=c$ where $a<c<b$. According to Theorem 5.2, the equation $M v=0$ has in this case a solution $v$ such that $k$ $(1 \leqq k \leqq n-1)$ of the functions $L_{0} v, \ldots, L_{n-1} v$ vanish at $x=a$ and $n-k$ at $x=c$. If $u$ is any solution of $L u=0$, it follows from (6.3) that

$$
\left[M_{0} v L_{n-1} u-M_{1} v L_{n-2} u+-\cdots-(-1)^{n} M_{n-1} v L_{0} u\right]_{a}^{c}=0 .
$$

Because of our choice of $v, n$ of the $2 n$ terms appearing in (6.4) vanish. $n-1$ additional terms can be made to vanish by taking $u$ to be a solution of $L u=0$ for which a suitable set of $n-1$ of the functions $L_{0} u, \ldots, L_{n-1} u$ vanish at either $x=a$ or $x=c$ (evidently, this has to be done in such a way that $L_{r} u=0$ at points at which $L_{n-r-1} v \neq 0$; to make things definite we may, moreover, assume that $u$ is chosen so as to make all the terms $M_{r} v L_{n-r-1} u$ vanish at $x=a$ ). This leaves us with one surviving term, say, $M_{m} v L_{n-m-1} u, x=c$. According to (6.4), this term must also be zero. Since, by Theorem 5.4, $M_{m} v \neq 0$ at $x=c$, we may conclude that $L_{n-m-1} u=0$ at this point. But this was not one of the $n-1$ boundary conditions imposed on $u$, and our argument thus shows that each of the functions $L_{k} u, k=0, \ldots, n-1$, vanishes at either $x=a$ or $x=c$. Hence, the operator $L$ must have in $(a, c]$ a focal point with respect to $x=a$. This contradicts our assumption that $L$ is disfocal in $(a, b)$, and thus establishes Theorem 6.1. It may be remarked here that our argument also shows (by interchanging the roles of $L$ and $M$ ) that the focal points of an operator and its adjoint coincide.

We next use Theorem 6.1 to prove a comparison theorem for disfocal operators which is analogous to Theorem 4.1. 
THEOREM 6.2. Let $p_{1}(x)$ and $p_{2}(x)$ be continuous, and let

$$
0 \leqq p_{1}(x) \leqq p_{2}(x) \text {. }
$$

If the equation

$$
L u \equiv L_{n} u-\lambda p_{2}(x) u=0, \quad \lambda= \pm 1,
$$

is disfocal, then the same is true of the equation

$$
L^{*} u=L_{n} u-\lambda p_{1}(x) u=0 .
$$

We denote by $M$ the adjoint to $L$ and we note that, by Theorem 6.1, $M$ is likewise disfocal. Using (6.6), (6.7), and Green's identity (6.3) (with proper change of notation), we have

$$
\begin{aligned}
\int_{a}^{c}\left(v L^{*} u-u M v\right) d x= & \lambda \int_{a}^{c}\left(p_{2}-p_{1}\right) u v d x+\int_{a}^{c}\left(v L_{n} u-(-1)^{n} u M_{n} v\right) d x \\
= & \lambda \int_{a}^{c}\left(p_{2}-p_{1}\right) u v d x \\
& +\left[M_{0} v L_{n-1} u-M_{1} v L_{n-2} u+-\cdots-(-1)^{n} M_{n-1} v L_{0} u\right]_{a}^{c}
\end{aligned}
$$

or, if $u$ and $v$ are solutions of $L^{*} u=0$ and $M v=0$, respectively

$$
\lambda \int_{a}^{c}\left(p_{1}-p_{2}\right) u v d x=\left[M_{0} v L_{n-1} u-+\cdots-(-1)^{n} M_{n-1} v L_{0} u\right]_{a}^{c} .
$$

Suppose now that equation (6.7) is not disfocal in $(a, b)$. As shown in the proof of Theorem 5.1, this implies the existence of a focal point $c=\zeta(a)$ in $(a, b)$ and of a corresponding extremal solution $u$, the latter having the property that each of the functions $L_{0} u, \ldots, L_{n-1} u$ vanishes at either $x=a$ or $x=c$, but nowhere in $(a, b)$. According to Theorem 5.4, each of these functions is different from zero at the other end of the interval. In particular, $L_{n-1} u \neq 0$ at either $x=a$ or $x=c$.

We now construct a solution $v$ of $M v=0$ for which $M_{1} v, M_{2} v, \ldots, M_{n-1} v$ vanish at those ends of the interval $(a, c)$ at which, respectively, the functions $L_{0} u, \ldots, L_{n-2} u$ do not vanish, and we note that, since $M$ is disfocal in $(a, b)$, we necessarily have $M_{0} v \neq 0$ (and thus $v \neq 0$ ) throughout the interval [a,c]. For this choice of $u$ and $v,(6.8)$ reduces to

$$
\lambda \int_{a}^{c}\left(p_{1}-p_{2}\right) u v d x=\left[M_{0} v L_{n-1} u\right]_{a}^{c} .
$$

Since, by (1.2) and (6.7),

$$
\rho_{n+1} \frac{d}{d x} L_{n-1} u=\lambda p_{1}(x) u, \quad p_{1}(x) \geqq 0,
$$

we have $\left.\operatorname{sgn} L_{n-1} u\right|_{x=a}=\operatorname{sgn}(-\lambda u)$ if $\left.L_{n-1} u\right|_{x=c}=0$ and $\left.\operatorname{sgn} L_{n-1} u\right|_{x=c}=\operatorname{sgn} \lambda u$ if $\left.L_{n-1} u\right|_{x=a}=0$. By Theorem 5.2, neither $u$ nor $v$ vanish in $(a, c)$, and it thus follows from (6.9) that

$$
\int_{a}^{c}\left(p_{1}-p_{2}\right)|u||v| d x=\left|M_{0} v L_{n-1} u\right|_{x=a}
$$


if $L_{n-1} u=0$ at $x=c$, and

$$
\int_{a}^{c}\left(p_{1}-p_{2}\right)|u||v| d x=\left|M_{0} v L_{n-1} u\right|_{x=c}
$$

if $L_{n-1} u=0$ at $x=a$. Since, by (6.5), $p_{1}-p_{2} \leqq 0$, both possibilities lead to contradictions. This completes the proof of Theorem 6.2. It may be remarked that a procedure similar to the one employed in the present case can be used to give an alternative proof for Theorem 4.1.

7. In this section we show that disfocal operators of the form (5.1) have the following additional property.

THEOREM 7.1. If an nth order operator $L$ of the form (5.1) is disfocal in $(a, b)$, and if $u$ is a nontrivial solution of $L u=0$, then the combined number of zeros of the functions $L_{0} u, \ldots, L_{n-1} u$ in $(a, b)$ cannot exceed $n-1$.

For operators of the form (5.1), we thus have $\zeta(a)=\zeta^{*}(a)$, where $\zeta^{*}(a)=\inf c$ for all $c$ such that for no solution $u$ of $L u=0$ the combined number of zeros of $L_{0} u, \ldots, L_{n-1} u$ in $(a, c)$ exceeds $n-1$. It is easy to show that Theorem 7.1 has the following consequence.

THEOREM 7.2. If equation (5.1) is disfocal in $(a, c)$, then no minor of the fundamental Wronskian matrix can vanish in $(a, c)$.

This goes considerably beyond the assertion of Theorem 5.1, which refers only to the principal minors. It should however be noted that the nonprincipal minors are not all positive. By means of a more detailed analysis of the situation it is not too difficult to obtain a criterion indicating which of the minors are positive and which are negative. Since we have no occasion to use this criterion, we shall neither state nor prove it.

That Theorem 7.2 is a corollary of Theorem 7.1 is an immediate consequence of the fact that the vanishing of a minor of order $k$ at $x=c$ is equivalent to the existence of a solution $u$ for which $n-k$ of the functions $L_{0} u, \ldots, L_{n-1} u$ vanish at $x=a$, and $k$ of these functions vanish at $x=c$.

The proof of Theorem 7.1 uses essentially the same ideas as the proof of Theorem 5.2, and we accordingly confine ourselves to a few brief indications. If $\zeta^{*}(a)$ is the number defined above, we note that evidently $\zeta^{*}(a) \leqq \zeta(a)$. As before, we select a "maximal" solution $u$ for which the total number of zeros of $L_{0} u, \ldots, L_{n-1} u$ which are located at either $x=a$ or $x=\zeta^{*}(a)$ is as large as possible. As in the proof of Theorem 5.2, this solution has the uniqueness property which makes it possible to apply Lemma 5.1. In the same way as before, it is then shown that $L_{m} u$ cannot change its sign in $\left(a, \zeta^{*}(a)\right)$ for $m=0, \ldots, n-1$. This also excludes even-order zeros of $L_{m} u$, since these correspond to points at which $L_{m+1} u$ changes its sign (if $m=n-1, m+1$ is to be replaced by 0 ). The possibility that $L_{m} u=0$ at both $x=a$ and $x=\zeta^{*}(a)$ is also excluded, since Rolle's theorem would in this case lead to a 
change of sign of $L_{n+1} u$ in $\left(a, \zeta^{*}(a)\right)$. Since the total number of zeros of the functions $L_{0} u, \ldots, L_{n-1} u$ in $\left[a, \zeta^{*}(a)\right]$ must be at least $n$, this shows that each of these functions must vanish at either $x=a$ or $x=\zeta^{*}(a)$. In view of the definition of $\zeta(a)$, we thus have $\zeta^{*}(a) \geqq \zeta(a)$. Hence, $\zeta^{*}(a)=\zeta(a)$, and Theorem 7.1 is proved.

\section{REFERENCES}

1. G. Frobenius, Über adjungierte lineare Differentialausdrücke, Journal für Mathematik 85 (1878), 185-213.

2. W. Leighton and Z. Nehari, On the oscillation of solutions of self-adjoint linear differential equations of the fourth order, Trans. Amer. Math. Soc. 89 (1958), 325-377.

3. A. Yu. Levin, Some questions regarding the oscillation of solutions of linear differential equations. Dokl. Akad. Nauk SSSR 148 (1963), 512-515 = Soviet Math. Dokl. 4 (1963), 121-124.

4. J. Mikusiński, Sur l'équation $x^{(n)}+A(t) x=0$, Ann. Polon. Math. 1 (1955), 207-221.

5. G. Pólya, On the mean-value theorem corresponding to a given linear homogeneous differential equation, Trans. Amer. Math. Soc. 24 (1922), 312-324.

Carnegie Institute of Technology, Pittsburgh, Pennsylvania 